\title{
IMAGINACIÓN Y VOLUNTAD: REPENSAR LA EDUCACIÓN DESDE LA HERENCIA HUSSERLIANA DE LA FENOMENOLOGÍA EIDÉTICA DE PAUL RICOEUR
}

\author{
Luz María Ascárate Coronel* \\ doi:10.11144/Javeriana.uph34-69.ivhr
}

\section{RESUMEN}

La influencia de la fenomenología husserliana en los inicios del pensamiento de Paul Ricœur, en donde este elabora una fenomenología de la voluntad, es un tema de común acuerdo entre los especialistas. A este respecto, una de las herencias husserlianas más evidentes de las descripciones fenomenológicas ricœurianas de lo voluntario y lo involuntario es el método de descripción eidética, que sitúa a la imaginación en un lugar privilegiado. La relación que puede encontrarse en estas reflexiones entre los temas de la imaginación y la voluntad, creemos, puede darnos una perspectiva novedosa para repensar las problemáticas de la educación en la época contemporánea. Así, en el presente artículo, luego de presentar algunas de estas problemáticas de la educación desde la perspectiva de Ricœur, buscaremos una posible respuesta a ellas desde aquel ámbito de la fenomenología ricœuriana en donde se cruzan los temas de la imaginación y la voluntad.

Palabras clave: educación; voluntad; imaginación; fenomenología; Paul Ricœur

\footnotetext{
Pontificia Universidad Católica del Perú, Lima, Perú - École des Hautes Études en Sciences Sociales, París, Francia.

Correo electrónico: luz.ascarate89@gmail.com

Para citar este artículo: Ascárate Coronel, L.M. (2017). Imaginación y voluntad: repensar la educación desde la herencia husserliana de la fenomenología eidética de Paul Ricœur. Universitas Philosophica, 34(69), pp. 91-104. ISSN 0120-5323, ISSN en línea 2346-2426. doi:10.11144/Javeriana. uph34-69.ivhr
} 


\title{
IMAGINATION AND WILL: RETHINKING EDUCATION WITH PAUL RICOEUR'S EIDETIC PHAENOMENOLOGY AND ITS HUSSERLIAN LEGACY
}

\author{
Luz María Ascárate Coronel
}

\begin{abstract}
The influence that Husserlian phenomenology has exerted on Paul Ricœur's initial works on a phenomenology of will is a topic where specialists have achieved a mutual agreement. One of the most evident proofs of Husserlian influence on Ricœur's phenomenological definitions of the voluntary and the involuntary is the method of eidetic description, which privileges imagination. We believe that the relationship found within these reflections regarding imagination and will, may give us a novel perspective for rethinking the problems of education in the Contemporary Age. Hence, this article will introduce some of these issues regarding education from Ricœur's perspective, after which we will outline a potential solution based on the scope of Ricœurian phenomenology, whereupon the topics of imagination and will intersect.
\end{abstract}

Key words: education; will; imagination; phenomenology; Paul Ricœur 
EN ESTE ARTÍCULO NOS UNIMOS al grupo de pensadores que han reflexionado acerca del sentido de la educación y de la pedagogía desde los recursos que ofrece la filosofía de Paul Ricœur. Nos referimos a autores tales como Peter Kemp (2010), Bernt Gustavsson (1996), Johannes van der Ven (1998), Franc Morandi (2000) y Maria Duffy (2009). Sin embargo, mientras tales propuestas han aprovechado los desarrollos ricœurianos sobre la mímesis, la identidad narrativa, la pequeña ética y la memoria, respectivamente, nosotros nos enfocaremos en la primera etapa del pensamiento de Ricœur, en la que este desarrolla una fenomenología de la voluntad. Con esto, daremos respuestas a problemas que Ricœur identifica en los textos críticos que le ha dedicado al tema de la educación.

Cabe resaltar que el aporte que buscamos situar en la filosofía de Ricœur corresponde al ámbito de las bases o los fundamentos del campo educativo y no a la constitución de pedagogías específicas que puedan tener lugar efectivamente en esta o aquella institución ${ }^{1}$. Una acotación previa sobre el ámbito en el que nos situamos aquí es necesaria. En la actualidad, nos encontramos con opiniones dispares entre los especialistas sobre si una disciplina, una ciencia o un campo que tenga como objeto a la educación puede representar un ámbito del conocimiento susceptible de fundamentación.

Sobre ello, Bernard Charlot (2011) sostiene que:

[...] los investigadores de nivel universitario que se encuentran en departamentos, institutos, facultades dedicadas a las ciencias de la educación, o en cursos de posgrado referidos a la educación, no están de acuerdo sobre la existencia de una disciplina específica llamada ciencias de la educación o educación (p. 8) $)^{2}$.

Frente a esta situación, para Charlot, tenemos tres posibilidades. La primera consiste en tomar lo que se ha llamado ciencias de la educación como una entidad de validez únicamente institucional que no tiene ni necesita una epistemología específica ni un ámbito de investigación específico. La segunda posibilidad

1 Una reflexión sobre la educación y las instituciones que aprovecha mucho de las perspectivas ricœurianas y sí va en esta dirección es la de Kemp (2011).

2 Todas las traducciones al español son nuestras. 
consiste en hacer referencia a una cultura común constituida por trabajos de investigación y por prácticas comunes y transdisciplinaress en un campo específico (por ejemplo, desde la antropología, la sociología, la psicología), y, a partir de ello, atender a los efectos sociales de estas prácticas, más que a sus bases teóricas. Esta posibilidad ha sido explorada por los investigadores del llamado "campo educativo", cuyo alcance se ha inspirado en la sociología de Bourdieu. De este campo se destacan los nombre de Eduardo Weiss (2003), Enrique Martín Criado (2008) y Javier Corvalán (2012).

La tercera posibilidad, para Charlot, consiste en dar una definición que pueda construir una identidad común entre los investigadores de este tema y los que mantienen prácticas relativas a la pedagogía en instituciones educativas, y buscar construir la especificidad de una disciplina que cuente con conceptos y métodos propios. Esta tercera posibilidad, en la que Charlot (2011) se sitúa, debe comprenderse "como una pregunta abierta de la que no puedo aún tener respuesta" (p. 10). No obstante, cree él, esta posibilidad responde a un grupo real de investigadores que, al apostar por esta vía, asumen el desafío de la construcción de una disciplina o de una ciencia de la educación y dirigen su investigación hacia esta dirección.

Es justamente esta tercera posibilidad la que asumimos nosotros al dirigirnos a las bases o a los fundamentos de la educación. Esta, al apelar a un ámbito del conocimiento que aún se encuentra en construcción, en cuanto a su epistemología se refiere, nos exige caminar por ella. Como dice Ricœur (1971), "aventurándonos más allá del simple diagnóstico y arriesgándonos a explorar sus posiblidades" (p. 46). No es gratuito que cuando Ricœur se refiere al ámbito de la educación afirme que es necesario reconocer el tono problemático de estas investigaciones, por lo que nos pide tomar sus propuestas al respecto "como un ejercicio de la imaginación” (Ricœur, 1971, p. 46). Teniendo esto en cuenta, en lo que sigue, en primer lugar, a modo de un ejercicio de la imaginación, daremos un diagnóstico crítico de los desafíos de la educación según la perspectiva de Paul Ricœur y, en segundo lugar, presentaremos una posible respuesta desde la herencia husserliana de su fenomenología de la voluntad.

Según Peter Kemp (2010), la importancia que ha tenido Ricœur en la reflexión sobre la educación no es gratuita, pues "él estuvo muy interesado en educación como un problema filosófico de formación, y, en junio de 1968, luego de que colapsó el sistema de la universidad francesa durante la revuelta estudiantil, 
comenzó a escribir una serie de artículos al respecto" (p. 181). Concordamos con Kemp en el hecho de que los análisis que desarrolla Ricœur en esta época pueden ser vistos como un punto de partida para comprender la relevancia del pensamiento de Ricœur con respecto al rol de la educación en el presente. Ello porque, si bien el colapso del sistema universitario educativo se dio específicamente en el contexto de la educación francesa de 1968, esto le sirvió de impulso para pensar en la educación en sentido universal.

En uno de sus artículos, Ricœur (1971) define la educación con respecto a las dos finalidades que persigue desde su fundación: de una parte, impulsar la búsqueda del saber o de la verdad, y, de otra parte, dotar a la nación de medios profesionales (o lo que es lo mismo, constituir un espacio social con base en diplomas incuestionables). En esa sociedad, que Ricœur caracteriza como "sociedad en crisis", ambas finalidades se encuentran contrapuestas. Así pues, esta última finalidad, según Ricœur (1971), está convirtiendo a la educación cada vez más en un poder que se enlaza a otros poderes, una agencia de reclutamiento condenada a ser dirigida por la demanda social, una imagen no criticada o incriticable, dada por sentado, de la distribución de roles sociales:

La crisis que se extiende a todo el mundo profesional es un tema particularmente sensible hoy en donde la sociedad global está golpeada por la incertidumbre y la duda respecto de los fundamentos sobre los cuales reposa: sometimiento a la tecnología sin referencia a las necesidades reales de las personas ni a las condiciones de una existencia verdaderamente humana, tanto personal como comunitariamente; concepción puramente cuantitativa del crecimiento económico; la configuración del consumismo por la publicidad según los mismos patrones cuantitativos que la producción; manipulación de la opinión pública por los líderes de la mass media (Ricœur, 1971, p. 50).

Sobre esta sociedad en crisis que implica una crisis general de las instituciones, a la vez de que conlleva a una pérdida de confianza en ellas, la educación debe responder a la producción de roles sociales, exigencia que es cada vez menos compatible con la idea originaria de la búsqueda de la verdad y del saber.

Frente a este diagnóstico de una sociedad en crisis, la fenomenología se presenta como la rehabilitación del ejercicio de fundar, que ha caracterizado a la filosofía desde sus inicios en Grecia. Con la fenomenología se reivindica, además, 
el valor irreductible de la experiencia de la subjetividad frente a los esquemas cientificistas de corte positivista. Según Abbagnano y Visalberghi (2010), esto puede ser aprovechado al tratarse del ámbito de la educación:

La fenomenología, reivindica el valor primario, irreductible de la auténtica experiencia humana, contra los esquemas de la ciencia cuya función es en último término práctica y utilitaria. [...] Esta primacía es el postulado sobre el que puede fundarse con mayor provecho una obra concreta de reconstrucción social y educativa enderezada a realizar la comprensión universal entre todos los hombres, independientemente de su raza o credo político (p. 634).

Esta misma motivación la encontramos en la fenomenología de la voluntad que desarolla Paul Ricœur. Cabe decir que, aunque no haya acuerdo entre los especialistas del pensamiento ricœuriano sobre el lugar y la importancia de la fenomenología con respecto a la totalidad de la obra de Ricœur, sí hay común acuerdo respecto de la importancia de la fenomenología para el primer periodo de su pensamiento, en el que se enmarca y se desarrolla, inspirada en los análisis husserlianos, esta fenomenología de la voluntad de corte eidético. A esto es a lo que llamamos en el título de este artículo una "herencia husserliana". En esta etapa Ricœur busca hacer frente a lo que ha denominado "crisis de la sociedad" desde el ámbito fenomenológico. La imaginación y la voluntad se hacen lugar en la comprensión particular que él desarrolla de la fenomenología, aunque su comprensión de la obra de Husserl en ese momento no era de ningún modo acabada ni acertada, puesto que no tenía acceso a la totalidad de su obra, como admite el mismo Ricœur. No obstante, parece ser que incluso para los intérpretes contemporáneos de Husserl, el tema de la imaginación es indesligable del de la fenomenología.

Según Elliot (2005),

el rol primordial de las presentaciones imaginativas para el análisis fenomenológico se muestra de modo evidente. [...] [E]sta posibilidad de la reducción imaginativa deriva, para Husserl, del potencial universal de la conciencia para convertir un acto posicional de la percepción [...] en un acto no posicional de la imaginación que 'suspende' la realidad de la cosa intuida (p. 54).

Justamente, en las Lecciones de 1905, Husserl (2002) afirma: "como puedo 'reflexionar en la fantasía' puedo también efectuar la reducción fenomenológica en la fantasía" (p. 206). Así mismo, en Ideas I (1950), Husserl señala que la imaginación es una modificación de neutralidad; y que, a pesar del carácter 
particular que presenta su tipo, esta tiene una significación universal que puede aplicarse a todas las vivencias y que juega un rol importante en la mayor parte de formas que adopta la conciencia. Se distingue, no obstante, de la modificación de neutralidad que es una modificación universal de toda toma de posición, de toda tesis, y que se relaciona con la epojé, aquella que suspende la tesis general de la actitud natural. Antes bien, la fantasía es la modificación de neutralidad aplicada a la presentificación posicional del recuerdo (Husserl, 1950, p. 371). Es necesario resaltar aquí que, para Husserl, una presentificación (Vergegenwärtigung) es un título general que tiene las modalidades del recuerdo, fantasía, empatía y conciencia de imagen, a diferencia de la presentación (Gegenwärtigung) que tiene una única modalidad: la percepción. La fantasía es aquí una presentificación que es, a su vez, modificación de otra presentificación: el recuerdo. La conciencia de imagen es una presentificación que se erige directamente sobre la percepción. No obstante, en Hua XXIII, tanto fantasía como conciencia de imagen son aludidas con el título de imaginación, a secas.

Además, podemos establecer un nexo entre la abstención de posición propia de la modificación de neutralidad y la modificación de inactualidad (Elliot, 2005, p. 55) si atendemos a las descripciones de la mencionada Hua XXIII: mi teoría implica que todo género de cogitationes se ordene bajo la distinción entre actualidad e inactualidad [...], que en cada género actualidad signifique toda toma de posición efectiva, y que haya la posibilidad de abstenerse de una toma de posición, colocarla fuera de circuito, en suma, efectuar la modificación de inactualidad (Husserl, 2002, pp. 354-355).

Adicional a lo anteior, Husserl (2002) afirma que "la inactualidad es paralela a la fantasía pura y conforma el concepto de la imaginación en que justamente la simple imaginación significa interrumpir la actualidad. Toda consciencia de imagen es parte de esto" (p. 355). Así pues, con la descripción de la modificación de inactualidad "Husserl parece decir que cada aspecto de la vida consciente que afirma la existencia empírica (cada acto posicional) permite idealmente una conversión al modo 'como si existiera' de la conciencia imaginativa” (Elliot, 2005, p. 57). Además, en Ideas I Husserl (1950) afirma que la esencia del flujo de la vivencia en un yo vigilante implica que la cadena ininterrumpida de cogitaciones sea constantemente rodeada por una zona de 
inactualidad, siempre susceptible a convertirse al modo de la actualidad, así como recíprocamente, de actualidad en inactualidad (p. 115).

Ahora bien, al referirnos, dentro del método fenomenológico, a la importancia de la imaginación, esta se hace evidente por su proximidad a la percepción, que es el "principio de principios" de la fenomenología. Como afirma Husserl (1977):

No podemos intentar realizar una fenomenología de la percepción en forma del todo adecuada y completa sin tomar en cuenta aquel fenómeno cercano a la percepción. Al tomar este fenómeno en consideración, aquello que hemos aprendido será iluminado, suplementado y enriquecido. Nuestro objetivo inmediato es pues el realizar una fenomenología de la imaginación (p. 1).

Con respecto a la reducción eidética, para Husserl (1950, p. 5), la imaginación es la que permite las universalizaciones eidéticas de las formaciones conceptuales que conllevan a aprehensiones adecuadas por medio de nuestra capacidad de ver directamente la esencia en dichas generalizaciones. En efecto, afirma en el cuarto parágrafo de Ideas I que "el conocimiento de la esencia es independiente de todo conocimiento sobre hechos" (Husserl, 1950, p. 24) y está ligado a intuiciones "meramente ficticias", por oposición a las intuiciones empíricas que se atañen a la existencia. Sobre esto, Ricœur dice, en un pie de página de su traducción, que "la función de ilustración de la imaginación no es despreciable: la ficción es el verdadero revelador de la esencia” (Husserl, 1950, p. 24, nota del trad. 2), y nos anuncia, aquí mismo, que en el parágrafo 70 Husserl dirá que la ficción es el elemento vital de la fenomenología, como de toda ciencia eidética. Justamente, en el parágrafo 70, Husserl (1950) afirma que "la libertad en la investigación de esencias exige necesariamente que operemos sobre el plano de la imaginación” (p. 226).

Incluso si Ricœur no comprendió completamente el sentido husserliano de la reducción trascendental ni de la constitución, creemos que el sentido de la fenomenología que desarrolló en el horizonte eidético de su Filosofía de la voluntad, inspirándose en Husserl, nos descubre aspectos importantes de la imaginación y de su relación con la fenomenología. Parece ser que aquellas reflexiones de Husserl, a las que hemos aludido, sobre la reducción eidética que se encuentran en Ideas I, libro comentado y traducido por Ricœur, tuvieron mucha influencia en este periodo de su pensamiento. Incluso, antes de realizar esta traducción, en su primera publicación filosófica, que realizó a los 26 años, titulada "La atención. Estudio fenomenológico de la atención y de sus conexiones filosóficas”, Ricœur (2013) 
presenta a la fenomenología como la salida de la crisis en tanto que apela al ámbito de la libertad humana. La imaginación es aquí el rostro epistémico de la libertad.

Y es en la realización de su fenomenología -que toma a la libertad como tema protagónico- en donde inscribe al método fenomenológico en el marco de la voluntad. De este modo, leemos en un artículo programático sobre su filosofía de la voluntad lo siguiente:

La fenomenología apuesta por esta discursividad primordial de toda vivencia que [...] sea implícitamente un "decir", un $\lambda \dot{\varepsilon} \gamma \varepsilon \imath v$; si la posibilidad de decir no estuviera inscrita en el "querer decir" de la vivencia, la fenomenología no

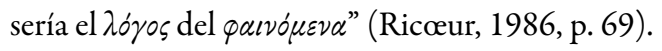

La proximidad del ejercicio del método fenomenológico al tema de la voluntad humana, para Ricœur (1950b), se relaciona, pues, directamente con el tema de la libertad. Esto es claro si atendemos a su descripción de la reducción. Así pues, en su introducción a la traducción de Ideas I de Husserl, afirma:

Me encuentro primero olvidado y perdido en el mundo, en las cosas, en las ideas, en las plantas y en las bestias, perdido en el otro, perdido en las matemáticas [...], hay en el ver una trampa, la trampa de la alienación [...]. Comprendemos así que el naturalismo sea el grado más bajo de la actitud natural [...] porque si me pierdo en el mundo, me encuentro ya a un paso de tratarme como cosa del mundo. La tesis de la actitud natural es una especie de cosidad en el sentido mismo del ver, aquello que se llamó vivir es aquí esconderme como conciencia ingenua en [...] la existencia de las cosas [...]. Así, la ascesis fenomenológica es una verdadera conversión del sentido de la intencionalidad que es primero olvido de una conciencia que luego se descubre como don (Ricœur, 1950a, p. xx).

Es por ello que, para Ricœur, la reducción es el primer gesto libre en tanto "liberador de la ilusión mundana" (p. xx). Es con ella que perdemos aparentemente el mundo que ganamos realmente.

En el primer tomo de su Filosofía de la voluntad, Ricœur (1950b) se decide, sin embargo, por utilizar la reducción eidética, mas no la trascendental. De esta última prefiere alejarse por ser tan "famosa" como "oscura" (p.7). Con la expresión "famosa", Ricœur pone énfasis en las diversas lecturas que hacen los lectores de Husserl en la época, que él prefiere no suscribir. Con la expresión "oscura”, muestra su actitud humilde frente a una comprensión cabal del método fenomenológico 
que le es vedada en ese momento de la historia. En cuanto a la dimensión eidética, aquello que se hace evidente es la importancia de la imaginación en el método:

En efecto, al poner entre paréntesis la culpa, que altera profundamente la inteligibilidad del hombre, y la trascendencia, que encubre el origen radical de la subjetividad, se constituye una descripción pura y una comprensión de lo voluntario y de lo involuntario [...]. Esta abstracción se emparenta [...] con aquello que Husserl ha llamado "reducción eidética", es decir, la puesta entre paréntesis del hecho y el aparecer de la idea, del sentido.

Las esencias del querer es lo que comprendo bajo un modelo imaginario, cuando digo: proyecto, motivo, necesidad, emoción, carácter, etc. Una comprensión esquemática de estas funciones claves precede a todo estudio empírico e inductivo llevado a cabo con métodos experimentales propios de las ciencias de la naturaleza. Es esta comprensión directa del sentido de lo voluntario y de lo involuntario lo que queremos aquí elaborar (Ricœur, 1950b, pp. 7-8).

En sus reflexiones sobre el método a ejercer, Ricœur hace una distinción entre descripción y explicitación: "el primer principio que nos ha guiado en la descripción es la oposición de método entre la descripción y la explicación. Explicar es siempre volver lo complejo, simple" (Ricœur, 1950b, p. 8). Aplicado a la psicología, esta regla que dirige a las ciencias de la naturaleza nos obliga a remitir lo voluntario a lo involuntario. Por el contrario, la descripción tiene como punto de partida la

reciprocidad de lo involuntario y lo voluntario: la necesidad, la emoción, el hábito, etc., toman sentido completo únicamente en relación con una voluntad que los solicita, los inclina, los afecta y fija su sentido, es decir, los determina por su decisión, los mueve por su esfuerzo y los adopta por su consentimiento (Ricœur, 1950b, p. 8).

Ricœur, pues, deriva lo involuntario de lo voluntario y no al revés, y es por ello que su descripción es comprensión.

Los análisis de Ricœur (1950b), entonces, se dividen en tres partes y cada parte comienza por una descripción de lo voluntario: la decisión (pp. 37-186), el acto y el desplazamiento (pp. 187-318), y el consentimiento (pp. 319-456). En la primera parte, donde Ricœur describe la atención voluntaria, se refiere a la imaginación como aquella que nutre al querer y le otorga las posibilidades de 
aprehensión de valores. A esto le llama "el carácter universalmente imaginizante de la atención" (Ricœur, 1950b, p. 143). Afirma aquí que "poner atención es ver en sentido amplio, no intelectualista, es decir, de cierto modo, desarrollar intuitivamente todas las relaciones y todos los valores" (Ricœur, 1950b, p. 143)3.

Además, la relación entre libertad e imaginación en el ámbito de la voluntad aparece en esta última parte, en la que Ricœur (1950b) posiciona a la esperanza como el rechazo del consentimiento ejercido por la libertad:

La admiración dice: el mundo es bueno, es la patria posible de la libertad; puedo consentir. La esperanza dice: el mundo no es la patria definitiva de la libertad; consiento lo más posible, pero espero ser liberado de lo terrible y, en el fin de los tiempos, disfrutar de un nuevo cuerpo y de una nueva naturaleza en un ámbito de libertad (p. 451).

En esta línea interpretativa, Marie France Begué (2002) nos dice, sobre la fenomenología de la imaginación de Ricœur, lo siguiente: "la imaginación se acompaña con la libertad. Este acompañamiento se da en dos niveles: el de liberar al hombre, en lo posible, del reino de la necesidad, y el de incentivar la afirmación y la promoción del sí-mismo" (pp. 26-27). Dicha liberación puede ser comprendida como un educar en sentido originario. Justamente, afirma Ricœur, en otra parte, que "nuestra labor de enseñar tiene éxito en la medida en que se libera al estudiante (é-ducation)" (Ricœur, 1948, s. n.). En ese sentido, si apelamos una vez más a ese ámbito fundamental de la educación al que hicimos alusión al inicio de esta presentación, podríamos comprender la labor del educar como un filosofar aplicado, como suscribe Suchodolski (1979).

En este sentido, la posibilidad de hacer frente a la crisis en la que nos encontramos, cree Ricœur, se encuentra en "limpiar" la educación y reubicar su centro de actividad en la búsqueda libre del saber y la verdad. Esto es posible, afirma, si reconciliamos libertad y poder, espontaneidad e institución. La razón de esto es que la educación se encuentra en una posición privilegiada frente a la crisis en el sentido de que sus desventajas pueden convertirse en ventajas: la educación, aunque pertenezca al ciclo de la reproducción del capital, "no es una empresa en el sentido económico de la palabra. Ciertamente, produce el saber, la competencia,

3 Es por esto que, en su reciente libro, Amalric (2013), defiende la tesis de que Ricœur se aleja del intelectualismo al colocar a la imaginación como aquella que guía el querer (vouloir). 
en suma, los roles sociales, pero no los produce bajo la forma de mero trabajo, del servicio diferido" (Ricœur, 1971, p. 54). La educación se produce de modo discordante y salvaguarda un espacio crítico, por lo que "es un lugar privilegiado para librar la lucha en contra de la burocracia [... y por la invención de nuevos modelos flexibles del poder en donde la espontaneidad y la institución estén mejor equilibradas" (Ricœur, 1971, p. 54).

La educación, pues, es una "de las raras instituciones en donde la crítica y la creación de los ideales pueden lograrse" (Ricœur, 1971, p. 56); con ello, hace posibles los objetivos de una fenomenología de la voluntad y la imaginación. A su vez, puede ser un laboratorio de ideales y motivaciones para la sociedad posindustrial. Por tanto, la función crítica y creadora de esta institución "debe ubicarse en el nivel de las cuestiones fundamentales, en el nivel más profundo de la dinámica social, y no en el plano de las alternativas políticas en el sentido partidista" (Ricœur, 1971, p. 56), en el nivel filosófico propiamente dicho.

De cualquier modo, Ricœur afirma que su intención no es presumir haber resuelto los problemas de la educación, sino comunicar la convicción de que estos problemas existen y que deben enfrentarse con una voluntad firme dispuesta a "desburocratizar", la cual se acompaña de una movilidad institucional y de un espíritu de invención en alerta. Ricœur (1971) afirma que "la 'vieja dama' [podemos comprender aquí 'la educación'] perecerá si, luego del fracaso de la imaginación en su intento por tomar el poder, el poder no toma a la imaginación” ( $p$. 58). Poder debe ser aquí comprendido como una expresión que nos remite a la voluntad del ser humano y abre paso al ámbito de la libertad.

Con todo, filosofía, fenomenología y educación pueden ser concebidas, desde aquí, como distintos modos de expresar una misma lucha contra la crisis burocratizante, la crisis de pérdida de sentido y de asentamiento de viejos dogmatismos. En ese sentido, en el contexto en el que nos encontramos, al interior de instituciones educativas con tensiones específicas entre un impulso humanista y una motivación productiva, puede ser provechoso plantear, desde la impronta ricœuriana, la siguiente hipótesis: quizá si la lucha frente a la crisis se sitúa en el cuestionamiento aunado del sentido originario de la filosofía, la fenomenología y la educación, ¿no podríamos abrirnos paso a un campo distinto de respuestas que impliquen repensar nuestras instituciones reales sobre la dinámica de la voluntad crítica y la creatividad imaginativa? 


\section{Referencias}

Abbagnano, N. \& Visalberghi, A. (2010). Historia de la pedagogia. (Trad. J. Hernández). México D. F.: Fondo de Cultura Económica.

Amalric, J. L. (2013). Paul Riccur, limagination vive. Une genèse de la philosophie riccurienne de l'imagination. Paris: Hermann.

Begué, M. F. (2002). Paul Ricour: La poética del sí-mismo. Buenos Aires: Biblos.

Charlot, B. (2006). A pesquisa educacional entre conhecimentos, políticas e práticas: especificidades e desafios de uma área de saber. Revista Brasileira de Educação, 11(31), 7-18. Recuperado de https://dx.doi.org/10.1590/ S1413-24782006000100002

Corvalán, J. (2012). El campo educativo: ensayo sociológico sobre su diferenciación y complejización creciente en Chile y América Latina. Estudios Pedagógicos, 38, 287-298. Recuperado de http://www.scielo.cl/pdf/estped/v38n2/ art18.pdf

Duffy, M. (2009). Paul Riccur's Pedagogy of Pardon. New York: Continuum.

Elliot, B. (2005). Phenomenology and Imagination in Husserl and Heidegger. New York: Routledge.

Gustavsson, B. (1996). Bildning $i$ vair tid. Stockholm: Wahlström \& Wiedstrand.

Husserl, E. (1950). Idées directrices pour une phénoménologie et une philosophie phénoménologique pures. Tome premier: Introduction générale à la phénoménologie pure. (Trad. P. Ricœur). Paris: Gallimard.

Husserl, E. (1977). Phenomenological Psychology. Lectures, Summer Semester, 1925. En Husserliana (Vol. IX). (Trad. J. Scanlon). The Hague: Martinus Nijhoff.

Husserl, E. (2002). Phantasia, conscience d'image, souvenir. En Husserliana (Vol. XXIII). (Trads. R. Kassis \& J. F. Pestureau). Grenoble: Jérôme Million.

Kemp, P. (2010). Ricœur and Education: Ricœur's Implied Philosophy of Education. En S. Davidson (Ed.), Riccur Across the Disciplines (pp. 181-194). New York: Continuum. 
Kemp, P. (2011). La universidad desde una perspectiva cosmopolita. Études Ricœuriennes/Ricœur Studies, 2(2), 118-128. Recuperado de http://ricœur. pitt.edu/ojs/index.php/ricœur/article/view/105

Martin Criado, E. (2008). El concepto de campo como herramienta metodológica. Revista Española de Investigaciones Sociológicas, 123, 11-33. Recuperado de http://www.reis.cis.es/REIS/PDF/REIS_123_011215166984248.pdf

Morandi, F. (2000). Philosophie de l'Éducation. Paris: Nathan.

Ricœur, P. (octubre, 1948). Comment respecter l'enfant?. Conferencia dictada en el Congrès de la Fédération Protestante de l'Enseignement, Glay, Francia.

Ricœur, P. (1950a). Introducción. En Husserl, E. (1950). Idées directrices pour une phénoménologie et une philosophie phénoménologique pures. Tome premier: Introduction générale à la phénoménologie pure (pp. xi-xxxix). Paris: Gallimard.

Ricœur, P. (1950b). Philosophie de la volonté 1 Le volontaire et l'involontaire, Paris : Aubier, 1950.

Ricœur, P. (1971). L'avenir de l'université. En L. Dion, E. F. Sheffield, \& P. Ricœur (Eds.), L'enseignement supérieur: bilans et prospective (pp. 46-58). Montreal: Presses de l'Université de Montreal.

Ricœur, P. (1986). Méthode et tâches d'une phénoménologie de la volonté. En $\grave{A}$ l'école de la phénoménologie (pp. 65-92). Paris: Vrin.

Ricœur, P. (2013). L’attention. Étude phénoménologique de l'attention et de ses connexions philosophiques. En O. Abel \& P. Marinescu Studia Phanomenologica (Vol. XIII) (pp. 21-50). Bucharest : Centenary, Humanitas.

Suchodolski, B. (1979). Philosophy and Education. Review of Education, 25(2-3), 347-366.

Van der Ven, J. A. (1998). Formation of the Moral Self. Cambridge: Eerdmans.

Weiss, E. (2003). Introducción. En E. Weiss (Coord.), El campo de la investigación educativa (1992-2002) (pp. 35-46). México, D.F: Consejo Mexicano de Investigación Educativa. Recuperado de http://www.comie.org.mx/ doc/portal/publicaciones/ec2002/ec2002_v01.pdf 